\title{
STUDY ON COMPRESSIVE STRESS RELAXATION BEHAVIOR OF BEECH BASED ON THE FINITE ELEMENT METHOD
}

\author{
Wen Gang Hu', Hui Yuan Guan ${ }^{2,-}$
}

\begin{abstract}
The compressive stress relaxation behaviors in three directions of beech (Fagus orientalis) were studied by experiments, and predicted by mechanical model and finite element method. Firstly, shortterm (3 hours) compressive stress relaxation experiments were carried out in longitudinal (L), radical (R) and tangential (T) directions of beech, and then the experimental data was fitted by mechanical model with two single Maxwell bodies in parallel connection. Secondly, the method of predicting the long-term (12 hours) stress relaxation behaviors of beech based on the finite element method was studied using the experimental data of short-term stress relaxation. Finally, the long-term stress relaxation behaviors in three directions of beech were investigated by experiments, mechanical model and finite element method respectively, and the results of them were compared. The results showed that stress relaxation behaviors of beech were different in three directions, and the short-term stress relaxation in $\mathrm{L}$ was much smaller than those in $\mathrm{R}$ and $\mathrm{T}$ directions under the same load. Besides, the mechanical model with two single Maxwell bodies in parallel connection was capable of predicting the short-term relaxation behaviors of beech in three directions with correlation coefficients beyond 0,99 but it did not work in long-term relaxation. In addition, the errors of FEM were smaller than those of the mechanical model compared with the results of experiments in the long-term stress relaxation, and the errors of the FEM were approximately $8 \%$ in L and $20 \%$ in R and T directions, which were all accepted in the field of wood engineer. This study will contribute to predict the long-term relaxation behaviors of wood products and wooden structures based on the FEM.
\end{abstract}

Keywords: Fagus orientalis, maxwell model, mechanical properties, modulus of elasticity, wood structures.

\section{INTRODUCTION}

Wood is a natural biomass material, which has complex physical and mechanical properties, including the elasticity of solid and of viscosity of fluid, so it is called viscoelastic material. Although creep and stress relaxation are two main parts of viscoelasticity, there is relation between them. The stress relaxation can be regarded as creep in different stress levels (Zhou et al. 2001). The viscoelasticity of wood plays an important role in durability of solid wood structures and wood products, which leads to decrease the strength of wood beam and wood joints (Figueroa et al. 2012, Daniela et al. 2013).

There are many factors influencing the viscoelasticity of wood, such as temperature (Peng et al. 2017a), humidity (Jiang et al. 2017) and stress levels (Peng et al. 2017b, Zhang et al. 2017). However, it is difficult to evaluate long-term viscoelastic behavior of wood for it is time consuming. Thus, many researchers have been studying how to predict the long-term creep and stress relaxation of wood by 
short-term experimental data. Empirical method (Mukudai et al. 1978, Mukudai and Sakamoto 1978) and mechanical method (Taniguchi et al. 2010, Chang et al. 2013) are two most used of them. The former used empirical equation to predict long-term viscoelasticity of wood, and the latter predicted viscoelastic behavior though using fitting curves of mechanical models, such as Maxwell, Kelvin, and Burgers. Hunt (2004) studied the method of using short-term creep data to evaluate long-term viscoelastic behavior of spruce in bending and beech in tension. In a carefully controlled creep experiment at constant humidity the results after the first few hours appear as a straight line, whether plotted as creep versus log time or as logarithm of creep rate versus creep. Then the curve was fitted and used to predict long-term creep behavior. Ozyhar et al. (2013) investigated the time dependency of the orthotropic compliance for beech wood by tensile and compressive creep experiments.

In fact, wood is usually used in a dynamic environment of temperature and moisture. Mechanosorptive (MS) creep is the deformation of loaded wood under changing climatic conditions. Salin (1992) studied on the dependence of the amount of checking on shrinkage induced stress level during timber drying with a new MS model and compared theoretically calculated maximal stress with observed amount of checking in a set of drying tests. Zhang et al. (2007) investigated the effects of temperature on MS creep of delignified hinoki wood (Chamaecyparis obtusa Endl.) using L and R specimens during adsorption and desorption over the temperature range of $20^{\circ}-80^{\circ} \mathrm{C}$. It was found that the effects of temperature on the MS creep of delignified specimens were more remarkable than for untreated specimens. Entwistle and Zadoroshny (2008) investigated MS strains which generated in strips of rectangular section stressed in torsion with the humidity cycled between $38 \%$ and $84 \%$. After a number of humidity cycles the specimen was unloaded, the applied stress was reversed and further humidity cycles were imposed, as well as all the initial strains were recovered and reversed strain was developed. It was concluded that the large MS strain produces no permanent change of the features of the cell wall structure that determine the shear modulus. There were close series between the mechanism generating the MS strains and that responsible for the large plastic strains (Keckes et al. 2003). Hering and Niemz (2012) analysed the pure creep behaviour of European beech wood in the longitudinal direction at three different moisture contents. The moisture-dependent creep compliance was identified using a four-point bending test device. The creep behaviour was ascertained to be linear with moisture content and quantified by means of a Kelvin-Voigt model approach. Huang (2016) carried out an experiment to investigate the influences of load and moisture content on MS creep of wood. It was found that the wood specimens exhibited a partial recovery during all the adsorption phase and deflection increase during all desorption phase when low load level was applied, and an amplified load effect existed within the creep under cyclic moisture changes.

With developments of computer science and finite element theories, FEM has been widely used in wood structures and wood products (Mackerle 2005). The studies on strength of wood mortiseand-tenon joint furniture based on FEM were investigated by $\mathrm{Hu}$ and Guan (2017a) and $\mathrm{Hu}$ et al. (2018a), but the viscoelasticity of wood were not considered. It is known to us the relaxation of wood joint will decrease the strength of joint significantly, and then influences the strength of the whole structure of wood products, since joints are the critical parts of wood structures. Beech wood is widely used in wood products in China. In this paper, the short-term stress relaxation behaviors of beech in $\mathrm{L}, \mathrm{R}$ and $\mathrm{T}$ directions were studied by experiments, and the data was analyzed by mechanical model and FEM respectively. Then the long-term relaxation behaviors were predicted by mechanical model and FEM, and the results were compared with those of long-term experiments. The aim of the study was to provide an efficient FEM to predict long-term stress relaxation of beech with short-term stress relaxation experimental data. This study will contribute to structure design of wood constructions and wood products based on FEM.

\section{MATERIALS AND METHODS}

\section{Materials and equipment}

The material used in experiment was beech (Fagus orientalis) wood bought from local wood commercial supplier (Nanjing, China). The specific gravity was $629 \mathrm{~kg} / \mathrm{m}^{3}$, and the moisture content 
was conditioned to and held at $10,8 \%$ before and during experiments. The main equipment used in the experiment was a $20 \mathrm{kN}$ universal testing machine (AGS-X, SHIMDZU, Japan).

\section{Specimen preparation}

All specimens used in this work were processed by computer numerical control machine with an accuracy of $0,01 \mathrm{~mm}$ (WPC, Yuli, China). The dimensions of specimen are $30 \mathrm{~mm}$ long $\times 20 \mathrm{~mm}$ wide $\times 20 \mathrm{~mm}$ thick, and the long directions are L, R and T respectively as shown in Figure 1. All specimens were cleared by sandpaper carefully before testing.

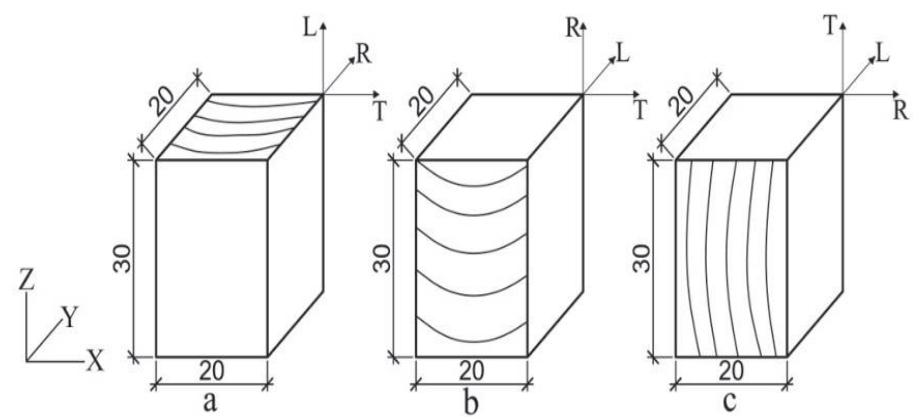

Figure 1: Dimensions of beech wood specimens in L (a), R (b) and T (c) directions.

\section{Test method}

An universal testing machine was used to load with loading rate $1 \mathrm{~mm} / \mathrm{min}$. The proportional limit loads in three directions of beech were measured in advance to make sure that the load imposed on specimens was smaller than the minimum proportional limit load of beech in three directions. After pretests, the proportional limit load of T direction was $1796 \mathrm{~N}$, which is minimum in three directions of beech wood. Thus, the load $1000 \mathrm{~N}$ was selected in this study to keep the specimens in elasticity when loaded.

Figure 2 shows the testing method of experiment. The specimens were compressed by steel loading head gradually till it reached $1000 \mathrm{~N}$, and then the displacement of steel loading head was kept as a constant for 3 hours. Ten times were repeated for each direction of beech. The stress relaxation behaviors were able to be recorded by transducer of universal testing machine, and then the data was analyzed by mechanical model and FEM respectively. Finally, the results of two methods were compared with that of long-term experiment.

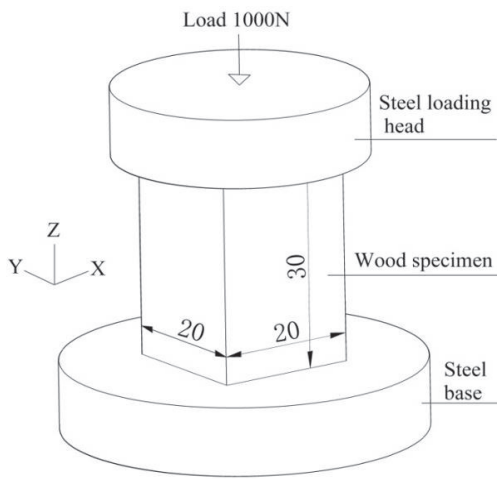

Figure 2: The setup to determine stress relaxation behaviors of beech. 


\section{Generalized Maxwell model}

A single Maxwell body can be regarded as a spring and a viscous damper in series connection, which is efficient in fitting the stress relaxation behavior. Figure 3 presents the generalized Maxwell model which is composed of multiple Maxwell bodies in parallel connection, and its mathematical expression is shown as Equation 1. In this study, the mechanical model is composed by two single Maxwell bodies connected in parallel, and Equation 1 can be fitted by Origin software (ref) based on the short-term experimental data.

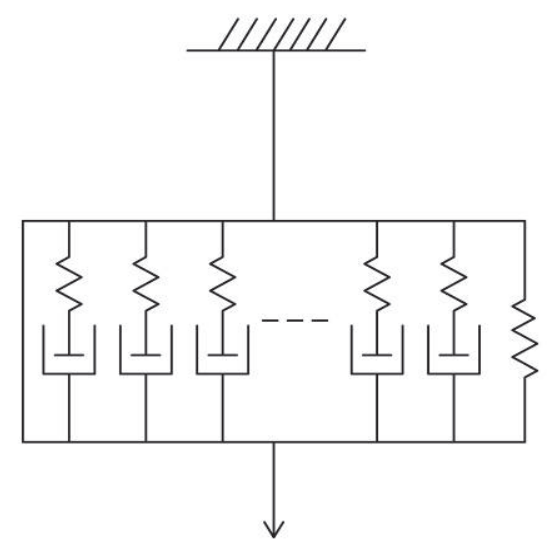

Figure 3: Diagram of the generalized Maxwell model.

$$
E_{(t)}=E_{(0)}+\sum_{i=1}^{n} E_{i} e^{-t / \tau}
$$

Where:

- $\boldsymbol{E}_{(t)}$ - refers to function of relaxation elastic modulus $\left(\mathrm{N} / \mathrm{mm}^{2}\right)$;

- $\boldsymbol{E}_{(0)}$ - is instantaneous elastic modulus $\left(\mathrm{N} / \mathrm{mm}^{2}\right)$;

- $\quad \boldsymbol{E}_{\boldsymbol{i}}$ - infers to elastic modulus of a Maxwell body $\left(\mathrm{N} / \mathrm{mm}^{2}\right)$;

- $\quad n$ - is the number of single Maxwell bodies, and $n$ is equal to 2 in this study;

- $\tau$ - refers to the ratio of viscosity to elastic modulus;

- $t$ - refers to time (s).

\section{Finite element method of stress relaxation}

In this paper, the ABAQUS 16.4-1(ref) was used to establish the finite element model. Generally, a finite element model includes following aspects, geometric model, mechanical properties of material, loads and constrains. In this paper, the stress relaxation parameters needed in ABAQUS were the key points to the study.

The geometric model is shown in Figure 1. The steel loading head and steel base were regarded as rigid, which were not deformation during loading process, and the beech specimen was loaded according to the procedures described in testing method of experiment. Besides, the specimen was meshed with C3D8 element, and totally 12000 elements were used in the model.

The elastic constants and yield stress of beech were measured in the previous study (Hu and Guan 2017b, Hu and Guan 2017c). For orthotropic beech wood, required inputs for elastic properties were 
three moduli of elasticity, three moduli of rigidity, and six Poisson's ratios as orthotropic material. The stress relaxation parameters of beech used in ABAQUS were the key. In ABAQUS, the visoelasticity was defined by a new constitutive equation expressed by Prony series parameters (Zhou et al. 2017) normalized shear modulus $\left(\mathrm{g}_{\mathrm{i}}\right)$ and parameter $\left(\tau_{\mathrm{i}}\right)$ shown in Equation 2.

$$
G_{(t)}=G_{(0)}+\left[1-\sum_{i=1}^{n} g_{i}\left(e^{-t / \tau_{i}}\right)\right]
$$

The methods of getting the parameters used in ABAQUS are as followings. Firstly, $F_{(t)}$ is the function of load and time during relaxation, which can be fitted by mechanical model with two single Maxwell bodies in parallel connection. Equation 3 is $F_{(t)}$ divided by $\mathrm{S}$ to get function of stress and time $\sigma_{(t)}$, and Equation 5 is the ratio of Equation 3 to Equation 4. Secondly, use Equation 6 to transfers $E_{(t)}$ to $G_{(t)}$. Finally, the Equation $7 G_{(t)}$ is expanded by Prony. The parameters $g_{\mathrm{i}}$ and $\tau_{\mathrm{i}}$ are what inputted into ABAQUS, which can be direved by Equation 8 and Equation 9.

$$
\begin{gathered}
\sigma_{(t)}=F_{(t)} / S(3) \\
\varepsilon=U / L(4) \\
E_{(t)}=\frac{\sigma_{(t)}}{\varepsilon}(5) \\
G_{(t)}=E_{(t)} /[2(1+u)] \\
G_{(t)}=G_{\infty}+\sum_{i=1}^{n} G_{i} e^{-t / \tau_{i}} \\
G_{0}=G_{\infty}+\sum_{i=1}^{n} G_{i}(8) \\
g_{i}=G_{i} / G_{0}(9)
\end{gathered}
$$

Where:

- $\quad \mathbf{F}_{(\mathrm{t})}$ - refers to the function of compression force $(\mathrm{N})$ and time (s)

- $\quad \mathbf{S}$ - is the area of cross section of specimen $\left(\mathrm{mm}^{2}\right)$

- $\sigma_{(t)}$ - infers as the function of stress $\left(\mathrm{N} / \mathrm{mm}^{2}\right)$ and time (s)

- $\varepsilon$ - represents strain

- $\mathbf{E}_{(\mathrm{t})}$ - refers to the function of elastic modulus $\left(\mathrm{N} / \mathrm{mm}^{2}\right)$ and time (s)

- $\mathbf{G}_{(\mathrm{t})}$ - refers to the function of shear modulus $\left(\mathrm{N} / \mathrm{mm}^{2}\right)$ and time (s)

- $\quad \mathbf{L}$ - refers to the length of specimen (mm)

- $\mathbf{U}$ - is the displacement (mm) when the load reached $1000 \mathrm{~N}$ 
- $\quad \mathbf{G}_{\infty}$ - is long term shear modulus $\left(\mathrm{N} / \mathrm{mm}^{2}\right)$

- $\mathbf{G}_{\mathbf{0}}$ - refers to instantaneous shear modulus $\left(\mathrm{N} / \mathrm{mm}^{2}\right)$

- $\mathbf{G}_{\mathrm{i}}$ - is relative shear modulus $\left(\mathrm{N} / \mathrm{mm}^{2}\right)$

- $\mathbf{g}_{\mathbf{i}}$ - infers as normalized shear modulus

- $\tau_{\mathrm{i}}$ - refers to relaxation parameter which is ratio of elastic modulus to viscosity

\section{RESULTS AND DISCUSSION}

\section{Experimental results of stress relaxation}

Figure 4 shows experimental results of compressive relaxation force $\mathrm{F}_{(\mathrm{t})}$ and relaxation modulus $\mathrm{E}_{(\mathrm{t})}$ in three directions of beech. They were fitted with mechanical model with two single Maxwell bodies in parallel connection by Origin software, and the correlation coefficients were all beyond 0,99. It suggested that two single Maxwell bodies in parallel connection were able to predict the short-term stress relaxation of beech in three directions. Figure 5 presents the comparison of compressive force and relaxation modulus in three directions. It shows that the compressive force in $\mathrm{L}$ direction decreases by $20 \%$, and those in $\mathrm{R}$ and $\mathrm{T}$ directions are $40 \%$ and $50 \%$ respectively, which indicates the stress relaxation of $\mathrm{L}$ direction was smaller than $\mathrm{R}$ and $\mathrm{T}$ directions at the end of tests. This phenomenon is opposite to that of Huc et al. (2018) who reported that wood experienced greater stress relaxation along the grain than perpendicular to it with the results based on 60min relaxation tests, which may cause by the effects of loading levels or time duration.

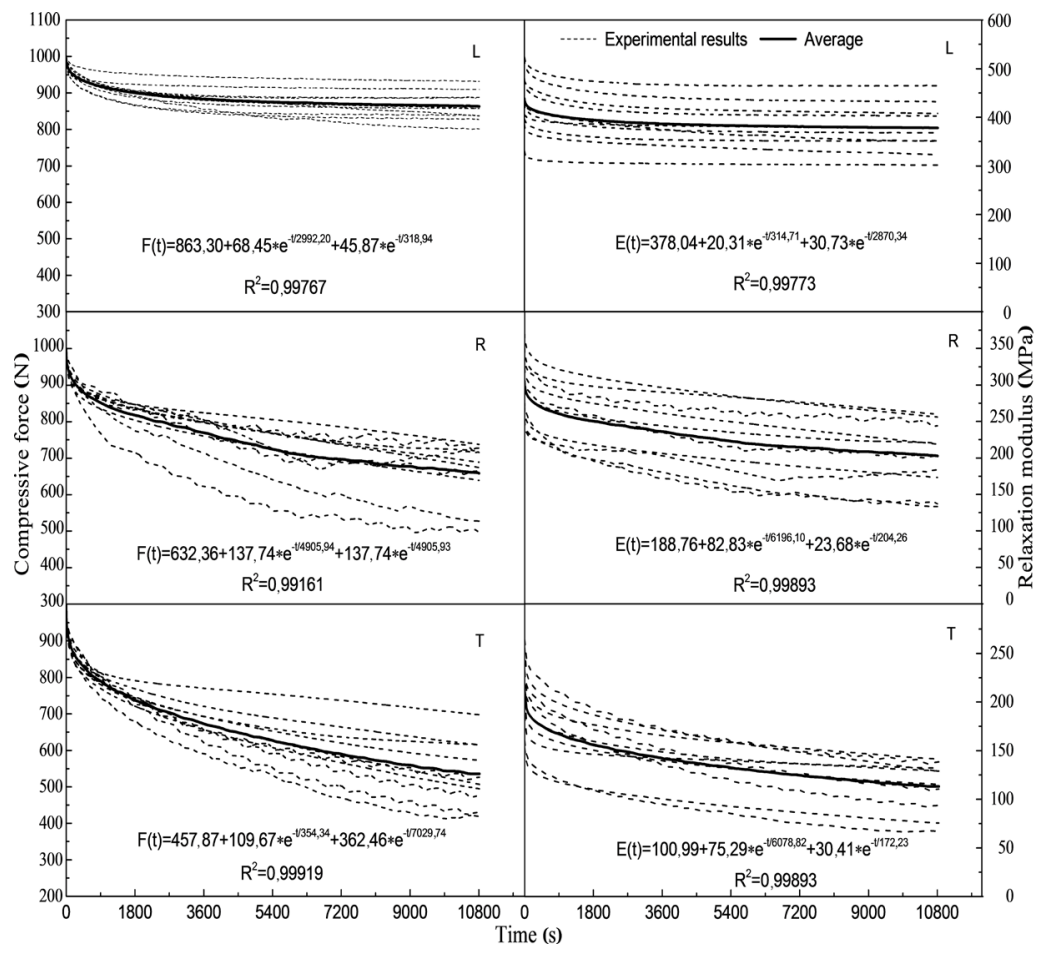

Figure 4: Curves of compressive force and time (left) and curves of relaxation modulus and time (right). 


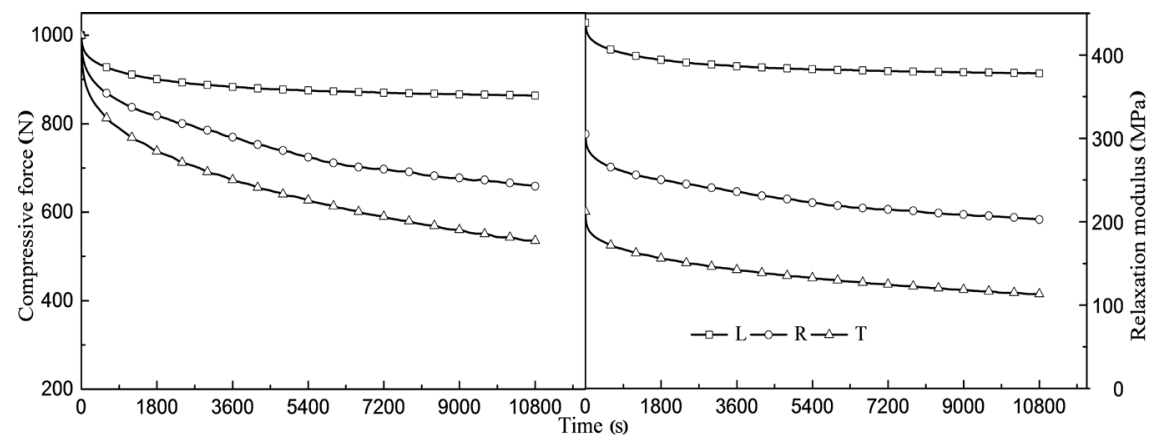

Figure 5: Compressive force and relaxation modulus in three directions of beech during 3 hours relaxation.

\section{Parameters used in ABAQUS}

Table 1 shows the parameters needed in ABAQUS in three directions of beech, which are figured out according to the calculating method described above. In this study, the second order $(n=2)$ Prony expansion parameters are used to predict the stress relaxation of beech in three directions.

Table 1: Dimensionless parameters used in FEM to simulate stress relaxation of beech.

\begin{tabular}{|c|c|c|c|}
\hline Directions & $\boldsymbol{n}$ & $\mathbf{g}_{\mathbf{i}}$ & $\mathbf{\tau}_{\mathbf{i}}$ \\
\hline \multirow{2}{*}{$\mathbf{L}$} & 1 & 0,047 & 314,713 \\
\cline { 2 - 4 } & 2 & 0,072 & 2870,335 \\
\hline \multirow{2}{*}{$\mathbf{R}$} & 1 & 0,281 & 6196,101 \\
\cline { 2 - 4 } & 2 & 0,080 & 204,264 \\
\hline \multirow{2}{*}{$\mathbf{T}$} & 1 & 0,318 & 6078,818 \\
\cline { 2 - 4 } & 2 & 0,158 & 172,231 \\
\hline
\end{tabular}

\section{Comparison and analysis}

Figure 6 shows the entire processes of stress relaxation behaviors of beech based on FEM. Figure $6 \mathrm{a}$ indicates the initial state without load imposed on the specimen, and Figure $6 \mathrm{~b}$ presents the state that the load reaches $1000 \mathrm{~N}$, as well as Figure $6 \mathrm{c}$ suggests the stress distributions of specimen decreases gradually during relaxation. Figure $6 \mathrm{~d}$ shows the stress distributions of specimens at the end of test. Obviously, for qualitative comparison, the stress relaxation behaviors of specimens are visualized base on the FEM, which can't be replaced by experiment. Besides, the results of compressive force are also able to be outputted by ABAQUS to compare with those of experiment quantitatively.

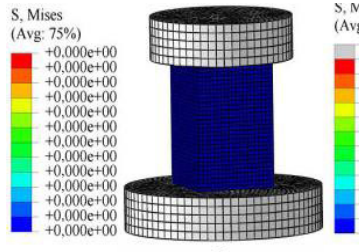

a

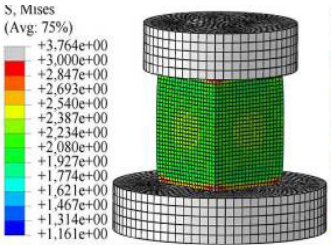

b

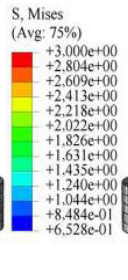

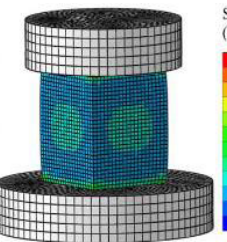

C

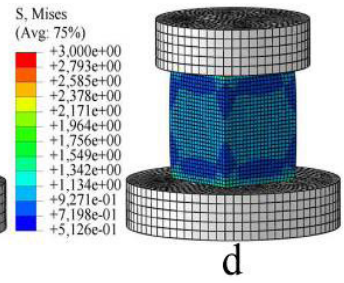

d

Figure 6: Stress distributions of specimen during the progresses of relaxation based on FEM. 
In the verification phase, long-term relaxation experiments in three directions of beech were determined by experimental method, and the mechanical model and FEM were also applied to predict the long-term relaxation using short-term experimental data. The compressive forces were extracted with one hour interval to compare with experiment. Relative errors between two methods (mechanical model and FEM) and experiment is shown in Figure 7. It indicates that the average errors of FEM are smaller than those of mechanical model, and the errors of FEM are within $8 \%$ in L direction and nearly $20 \%$ in $\mathrm{R}$ and $\mathrm{T}$ directions.

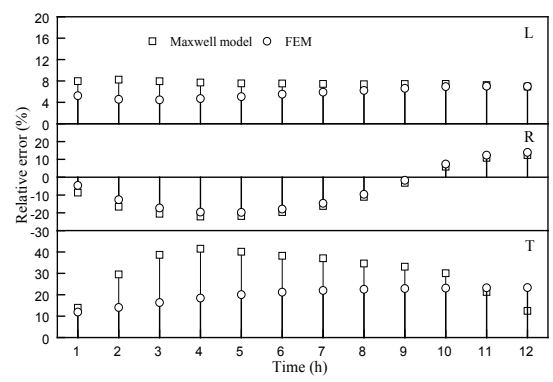

Figure 7: Relative errors between two methods (mechanical model and FEM) and experiment.

The aim of paper is to put forward a method of predicting the long-term stress relaxation with short-term experimental data. Compared with other methods, the FEM can present the processes of stress relaxation intuitively, and the accuracy is higher. Besides, the mechanical properties of small specimen can be used to analyze the stress relaxation of wood joint made by the same material based on FEM (Hu et al. 2018b). Although only the effect of directions of beech on stress relaxation behaviors were considered in this study, this method can also be used in other factors that influencing the stress relaxation of wood, such as temperature and humidity. In this work, only 3 hours experimental data was used to predict 12 hours relaxation by FEM, since the time and equipments were limited. The longer the relaxation time is, the more obvious the advantage of the FEM is. Two orders Prony expansion parameters (Table 1) were enough in ABAQUS to predict 12 hours relaxation in this paper. However, the longer the relaxation time is, the more orders Prony expansion parameters are needed.

\section{CONCLUSIONS}

The method of using FEM to predict long-term stress relaxation of beech with short-term relaxation data was studied, and compared with mechanical model and experiment. The following conclusions were drawn.

The stress relaxation of beech in $\mathrm{L}$ direction is smaller than those in $\mathrm{R}$ and $\mathrm{T}$ directions, and the compressive force decreases by $20 \%$ in $\mathrm{L}$ and $40 \%$ and $50 \%$ in $\mathrm{R}$ and $\mathrm{T}$ directions respectively.

The mechanical model with two single bodies in parallel connection is able to predict the shortterm stress relaxation of beech, but the errors increased as the time went by.

Relative error of FEM is smaller than those of mechanical model, and the error of FEM are within $8 \%$ in L direction and nearly $20 \%$ in $\mathrm{R}$ and $\mathrm{T}$ directions.

In conclusion, the stress relaxation behaviors of beech in three directions are different from each other, and the relaxation behavior of wood must be considered in wood products and wood constructions. The FEM is able to predict the long-term stress relaxation of beech with short-term experimental data, which will contribute to the structure design of wood products and wood constructions. However, 
further studies are also supposed to be conducted, such as the FEM to simulate the influences of temperature, humidity and load levels on the relaxation.

\section{ACKNOWLEDGMENTS}

This paper was supported by A Project Funded by Priority Academic Program Development of Jiangsu Higher Education Institutions (PAPD).

\section{REFERENCES}

Chang, F.C.; Lam, F.; Kadala, J.F. 2013. Application of time-temperature-stress superposition on creep of Wood-plastic composites. Mechanics of Time-dependent Materials 17(3): 427-437.

Sepulveda, D.; Bustos, C.; Gacitua, W.; Dechent, P.; Clouter, A. 2013. Effect of thermohygromechanical behaviour at macroscale of wood under compression perpendicular to the grain in nanomechanical properties of cellular structure. Maderas-Ciencia y Tecnologia 15(2): 205-222.

Entwistle, K.M.; Zadoroshny, A. 2008. The recovery of mechano-sorptive creep strains. Journal of Materials Science (43): 967-973.

Figueroa, M.; Bustos, C.; Dechent, P.; Reyes, L.; Cloutier, A.; Giuliano, M. 2012. Analysis of rheological and thermo-hygro-mechanical behaviour of stress-laminated timber bridge deck in variable environmental conditions. Maderas-Ciencia y Tecnologia 14(3): 303-319.

Hering, S.; Niemz, P. 2012. Moisture-dependent, viscoelastic creep of European beech wood in longitudinal direction. European Journal of Wood and Wood Products (70): 667-670.

Hu, W.G.; Guan, H.Y. 2017a. Experimental and numerical study on optimization design of stretcher positions. Wood Research 62(4): 575-586.

Hu, W.G.; Guan H.Y. 2017b. Study on elastic modulus of beech in different stress states. Journal of Forestry Engineering 2(6): 31-36.

Hu, W.G.; Guan, H.Y. 2017c. Investigation on withdrawal capacity of mortise and tenon joint based on friction properties. Journal of Forestry Engineering 2(4): 158-162.

Hu, W.G.; Guan, H.Y.; Zhang, J.L. 2018a. Finite element analysis of tensile load resistance of mortise-and-tenon joints considering tenon fit effects. Wood and Fiber Science 50 (2): 121-131.

Hu W.G.; Wan H.; Guan H.Y. 2018b. Study on contact force relaxation behavior of mortiseand-tenon joints considering tenon fits and grain orientations of tenon. Bioresource 13(3): 5608-5616.

Huang, Y. 2016. Creep behaviour of wood under cyclic moisture changes: interaction between load effect and moisture effect. Journal of Wood Science 62: 392-399.

Huc, S.; Hozjan, T.; Svensson, S. 2018. Rheological behavior of wood in stress relaxation under compression. Wood Science and Technology 52: 793-808.

Hunt, D.G. 2004. The prediction of long-time viscoelastic creep from short-time data. Wood Science and Technology 38(7): 479-492.

Jiang, J.L.; Bachtiar, E.V.; Lu, J.X.; Niemz, P. 2017. Moisture-dependent orthotropic elasticity and strength properties of Chinese fir wood. European Journal of Wood and Wood Products (75): 927938.

Keckes, J.; Burgert, I.; Fruhmann, K. 2003. Cell-wall recovery after irreversible deformation of 
wood. Nature Material (2): 810-813.

Mackerle, J. 2005. Finite element analyses in wood research: a bibliography. Wood Science and Technology 39(7): 579-600.

Mukudai, J.; Sakamoto, S.; Kadita, H.; Yata, S. 1978. Evaluating of linear viscoelastic behaviour of wood I. Mokuzai Gakkaishi (24): 447-454.

Mukudai, J.; Sakamoto, S. 1978. Evaluating of linear viscoelastic behaviour of wood II. Mokuzai Gakkaishi (24): 605-611.

Ozyhar, T.; Hering, S.; Niemz, P. 2013. Viscoelastic characterization of wood: Time dependence of the orthotropic compliance in tension and compression. Journal of Rheology (57): 699-717.

Peng, H.; Jiang, J.L.; Liu, J.X.; Cao, J.Z. 2017a. Application of time-temperature superposition principle to Chinese fir orthotropic creep. Journal of Wood Science 63(5): 455-463.

Peng, H.; Lu, J.X.; Jiang J.L.; Cao, J.Z. 2017b. Longitudinal mechano-sorptive creep behavior of Chinese fir in tension during moisture adsorption processes. Materials 10(8):931.

Salin, J.G. 1992. Numerical prediction of checking during timber drying and a new mechanosorptive creep model. European Journal of Wood and Wood Products (50): 195-200.

Taniguchi, Y.; Ando,K.; Yamamoto, H. 2010. Determination of three- dimensional viscoelastic compliance in wood by tensile creep test. Journal of Wood Science (56): 82-84.

Zhang, W.; Tokumoto, M.; Takeda, T. 2007. Effects of temperature on mechano-sorptive creep of delignified wood. Journal of Wood Science (53): 187-191.

Zhang, W.G.; Jiang W.Z.; Tang, R.Q. 2017. Study on short-term bending creep behavior and microstructure of bamboo scrumber. Journal of Forestry Engineering 2(3): 33-37.

Zhou, W.; Xie, F.X.; Zhang, C.J.; Ji, B.H.; Chen, L. 2017. Viscoelastic parameters determination method of pouring type asphalt mixture based on modified Burgers model. Journal of Forestry Engineering 2(3): 143-149.

Zhou, Z.G.; Qian, G.P.; Zheng, J.L. 2001. Research on the method o testing viscoelastic parameters of bituminous mixture. Journal of Chinese Communications University 17(4): 23-28. 